\title{
OSCILLATIONS IN PHOTODETACHMENT CROSS SECTIONS FOR IONS
}

\section{IN MAGNETIC FIELDS ${ }^{1}$}

Dakley H. Crawford

Heal th and Safety Research Division, Oak Ridge National Laboratory,
Oak Ridge. Tennessee 37831-6123

\section{ABSTRACT}

The photodetachment cross section of a charged particle bound in a short range potential is an oscillating function of frequency of incident light, in the presence of a magnetic field. The theory of this effect is described, and calculated cross sections are shown. For photodetachment of electrons from negative atomic ions, this constitutes an additional magnetic field effect beyond the ones associated with fine and hyperfine structure of the ion and atom and with spin of the detachedilectron.

\section{INTRODUCTION}

Electron detachment by laser in a magnetic field is of interest as a possible method for neutralizing negative ion beams. For example, Hershcovitch and Hinds ${ }^{1}$ have proposed a laser photodetachment scheme to generate a spin-polarized proton beam, taking advantage of the final-state interaction of the atom with a magnetic field. Also, we suggest that a magnetic field might improve the efficiency of photodetachment, due to a cross section enhancement arising from the interaction between the freed electron and the field. The theory of the effects on the photodetachment process of this electron-field interaction will be described here. A novel experimental approach to measurement of these effects is discussed in the following paper. 2

Magnetic-field-dependent structure in the photodetachment cross section of a negative ion was first observed by Blumberg. Jopson, and Larson. 3 Performing high resolution photodetachment spectroscopy on sulfur negative ions in an ion trap, they discovered oscillations in the cross section versus laser frequency, with a period proportional to the magnetic field strength. Blumberg and coworkers $^{3.4}$ attributed these features to detachment into a series of Landau states of the electron in the magnetic field.

A charged particle in a magnetic field has an infinite set of quantized energy levels (Landau levels) for motion perpendicular to

\footnotetext{
1 Research sponsored jointly by the U.S. Air Force Office of Scientific Research, under Interagency Agreement DOE No. 40-1262-82 and the Office of Health and Environmental Research. U.S. Department of Energy, under contract DE-ACO5-840R21400 with Martin Marietta Energy Systems. Inc. 
the field (while motion parallel to the field is unquantized). ${ }^{5}$ The spacing between these levels is $\hbar \omega_{H}$, where $\omega_{H}=2 \pi v_{H}=e H / m c, v_{H}$ being the cyclotron frequency and $H$ the magnetic field.

As a consequence, there is an infinite series of thresholds, separated by energy $\hbar \omega_{H}$. for detachment of an electron in a magnetic field. The theory of Blumberg et al. predicts that the cross section $\sigma_{n}$ for photodetachment into the $n$th Landau state is infinite at the threshold frequency. $v_{n}$ and decreases as $\left(v-v_{n}\right)^{-1 ;}$ for laser frequency $v>v_{n}$ : The total photodetachment cross section, obtained by suming the $\sigma_{n}$. thus consists of a series of infinite spikes. However, the result of convoluting over a distribution of Doppler shifts (to account for thermal motion) is finite. Using as free parameters the scale of the cross sections, the origin of the frequency scale, and the iōn temperature, a good $\mathrm{fit}^{4}$ to experiment was achieved.

Subsequent work has shown the importance of incorporating the final state interaction, ${ }^{6-10}$ i.e., between the electron and the atom into the theory. It has been proven ${ }^{7.9}$ that the cross section remains finite, given this interaction.

Recently, Gurvich and Zil 'bermints ${ }^{\circ}$ presented a detailed theory for photodetachment of a charged particle bound to a small-radius center in a magnetic field. Although motivated by experiments on $D^{-}$ and $\mathrm{A}^{+}$centers in semiconductors, this theory is applicable to the problem of interest here, as well. The treatment of Gurvich and Zil'bermints has been extended ${ }^{11}$ to handle arbitrary values of angular momentum and arbi yary laser polarization. Below. theoretical results for some cases of interest will be displayed and discussed.

\section{THEORY}

Consider a particle of charge -e bound to a potential of radius $r_{o}$, beyond which its value is negligible. We choose initial angular momentum and laser polarization to be such that dipole selection rules (ignoring the field) would determine unique values, ${ }^{12} \ell$ and $\mathrm{m}$. of the particle's final angular momentum quantum numbers, where $m$ is $^{13}$ the component in the direction of the magnetic field. The charged particle of interest is an electroin, but its spin does not enter into the theory of the effect under consideration here. The interaction of the spin with the magnetic field simply shifts the cross section in frequency.

Following Gurvich and Zil'bermints, 9 we assume

$$
\mathrm{kr}_{0} \ll 1,\left|\beta_{\ell}\right| \ll 1 \text {, }
$$

where 


$$
\beta_{\iota}=-2^{-1 / 2}(2 \iota+1) ! ! a_{\iota} a_{H}^{-2 \ell-1}
$$

where $k$ is the wavenumber of the electron and $a_{\ell}$ the scattering length, both in the final state, and $a_{H}=\omega_{H}^{-1 / 2}$ is the cyclotron radius of the electron. The second condition in (1) is satisfied when the scattering length exists, thus excluding a zero-energy bound state, and when the magnetic field is correspondingly not too large.

Now, consider the case $|m|=l$. This holds, for example, for detachment into an $s$ wave or a $P_{ \pm 1}$ wave. ${ }^{12}$ One finds that the cross section $\sigma^{\mathrm{H}}(\mathrm{knm})$ for photodetachment into the Landau level with quantum numbers $n$ is is given approximately by

$$
\sigma^{H}(\mathrm{knm})=\alpha(2 \iota+1) ! ! 2^{-1 / 2} a_{\mathrm{H}}^{-2 \iota-1}|M|^{2}\left[\begin{array}{c}
\iota+\mathrm{n} \\
\mathrm{n}
\end{array}\right]\left[\frac{\omega-\omega_{\mathrm{mn}}}{\omega_{\mathrm{H}}}\right]^{-1 / 2} \cdot|\mathrm{m}|=\iota
$$

where

$$
M=\left[1-i \beta \sum_{\iota} \sum_{n^{\prime}}\left[\begin{array}{c}
\ell+n^{\prime} \\
n^{\prime}
\end{array}\right]\left[\frac{\omega-\omega_{m n^{\prime}}}{\omega_{H}}\right]^{-1 / 2}\right]^{*-1}
$$

where $\omega_{m n}$ is the threshold frequency times $2 \pi$.

$$
\omega_{m n}=A+(n+b / m \mid+\not / m+1 / z) \omega_{H}
$$

A being the (field-free) binding energy in the initial state. The sum in Eq. (4) goes from $n=0$ to the first value of $n$ for which $\omega-$ $\omega_{\mathrm{mn}}<0$. The constant $\alpha$ in Eq. (3) is defined as the proportionality factor between the wavenumber and the cross section $\sigma(\mathrm{k} l)$ in the absence of a magnetic field.

$$
\sigma(\mathrm{k} l)=\alpha \mathrm{k}^{2 \ell+1} \fallingdotseq
$$

These equations are equivalent to the results of Gurvich and Zil'bermints. ${ }^{9}$ then written in the above form, they remain valid for two- or multi-photon processes, provided intermediate states are not affected by the magnetic field.

Fig. 1 shows a calculation for detachment into an $s$ state, taking $a_{\iota}=\alpha=1$. This result is relevant to photodetachment of $\mathrm{s}^{-}$. Of course, the latter would be modeled by a superposition ${ }^{3,4}$ of curves such as shown here, of differing magnitudes and threshold 


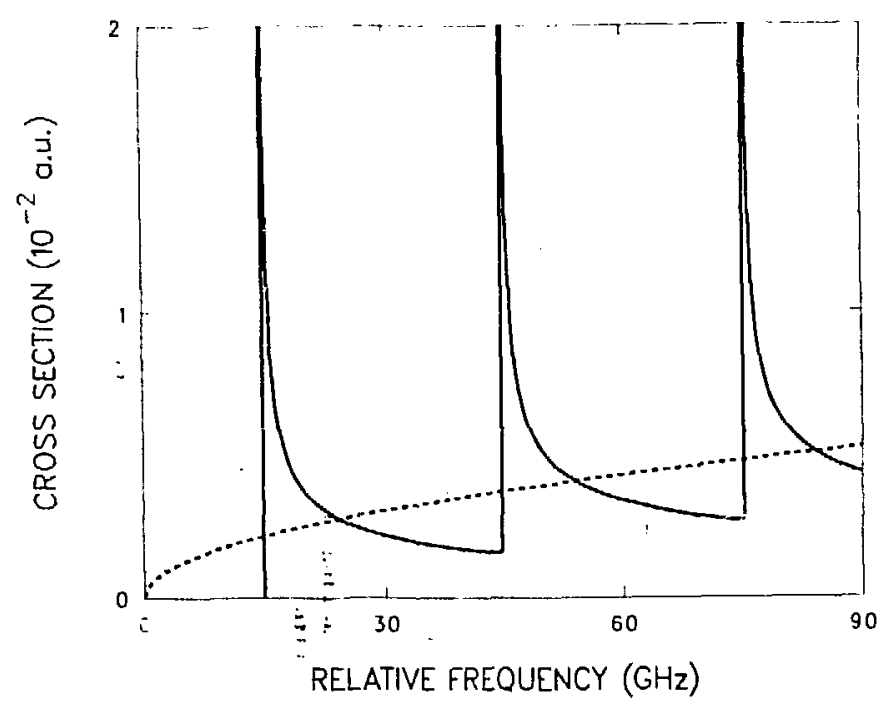

FIG. 1. Cross sections for photodetachment into an $s$ state in a $1.07 \mathrm{~T}$ magnetic field (solid curve) and in zero field (dashed curve), as functions of relative frequency of incident light. The frequency here and in Figs, 3 and 4 is measured relative to the threshold for $\mathrm{H}=0$ photode tachment.

frequencies due to the presence of fine structure in both the ion and the atom, and the spin of the detached electron. The solid curve is calculated for a magnetic field of $1.07 \mathrm{~T}$ (where $v_{\mathrm{H}}$ is 30 $\mathrm{GHz}$ or $\omega_{\mathrm{H}}=4.56 \times 10^{-6}$ a.u.) and the dashed curve is for zero field.

The solid curve in Fig. 1 consists of a series of sharp spikes appearing identical to the $\mathrm{k}^{-1}$ singllarities predicted by Blumberg et al. ${ }^{3}$ This is also the behavior predicted by Eq. (3) when $M=1$. From Eq. (4) it follows that $M$ is identically unity when the potential vanishes, in agreement $w i$ th the ofiginal theory. ${ }^{3.4}$ Also, $M \approx 1$ when, for all $\mathrm{n},\left|\omega-\omega_{\ell n}\right| \omega_{H} \gg \beta_{l}^{2}$. Here, $a_{H}=468$ a.u.. and $\beta_{\ell}=-1.51 \times 10^{-3}$ a.u., so the major departures from $k^{-1}$ behavior occur within only narrow regions, defined by $\left|\omega-\omega_{\iota n}\right| \lesssim 2.3 \times 10^{-6} \omega_{H}=68 \mathrm{kHz}$, around each threshold. 


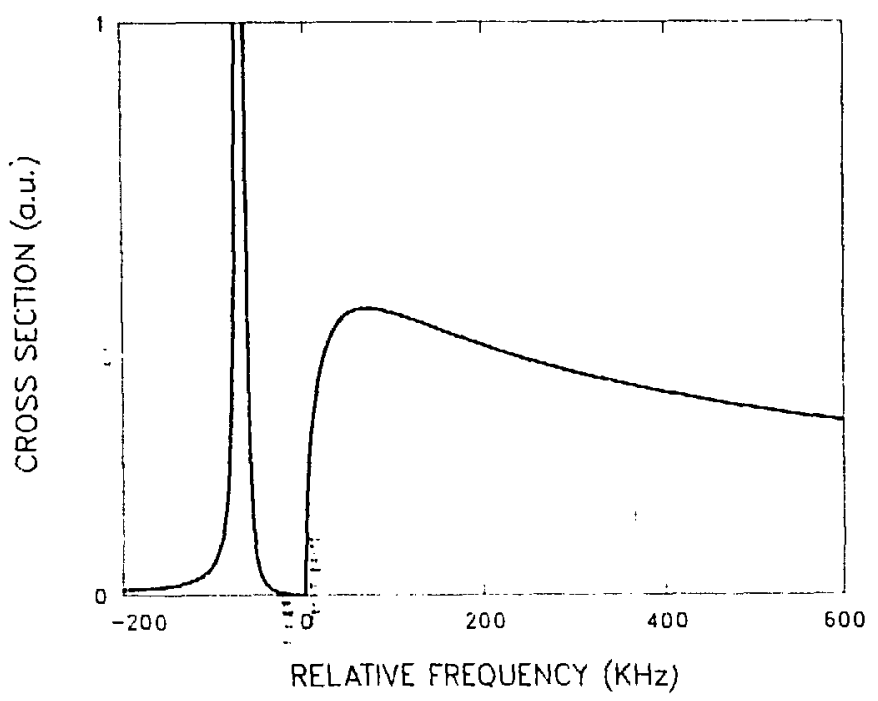

FIG. 2. Cross section for photodetachment into an s state in a $1.07 \mathrm{~T}$ magnetic field, as a function of relative frequency of incident light. The frequency is measured relative to the $n=1$ threshold.

The details of $\sigma$ near the $n=1$ threshold are shown in Fig. 2 . This is part of the cross section curve displayed in Fig. 1, but now on a greatly expanded frequency scale. Here, zero relative frequency denotes the threshold. We see peaks on both sides of threshold, with maxima at $\omega-\omega_{l n}= \pm \beta_{0} \omega_{H}= \pm 68 \mathrm{kHz}$.

The above features of the cross section have the following explanation. The Hamiltonian in the $\mathrm{n}=1$ channel has, whenever $\beta_{l}>0$, a bound state, of (very small) binding energy $\beta_{l} \omega_{H}$, in its spectrum, when coupling to the $n=0$ channel is ignored. That state manifests itself in two ways, First, because of the channel coupling, a resonance appears in the $n=0$ channel (at the same total energy), which shows up in Fig. 2 as the very sharp peak below the $n=1$ threshold. Second, the $n=1$ cross section becomes very large just above its threshold, just as ido elastic s-wave cross sections when a barely bound state exists.

The nature of the resonances below thresholds is clarified by considering that, in the final state wave functions, the $x$ and $y$ coordinates (perpendicular to $\mathrm{H}$ ) play the role of internal degrees of freedom. The problem becomes a one-dimensional multichannel one. (This representation is central to some of the previous work, and is implicit in the above discussion of coupled channels.) The features below thresholds are then identified as core-excited (Feshbach) resonances. These resonances occur below all but the lowest 


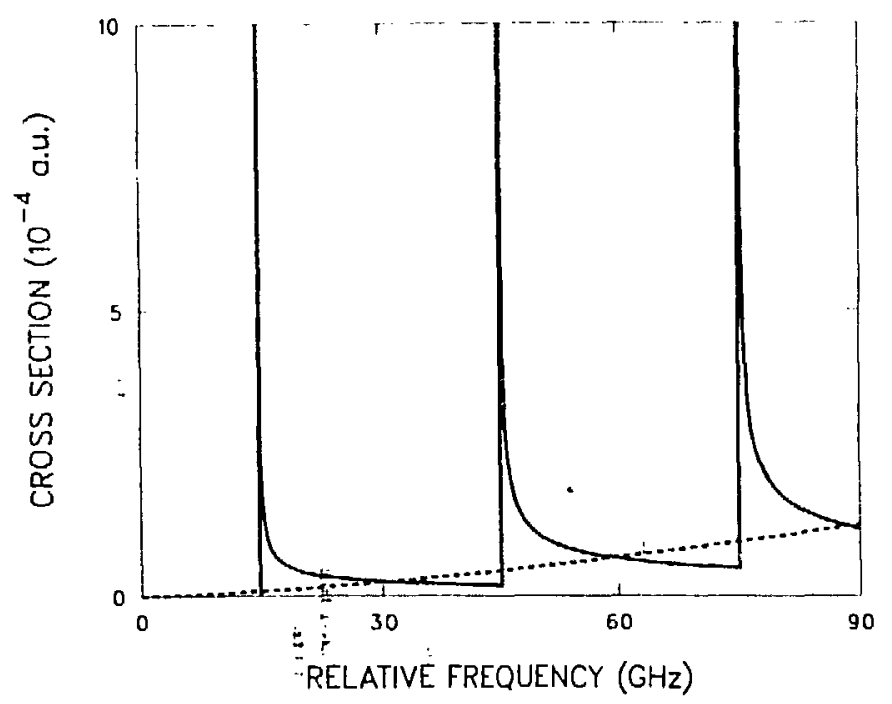

FIG. 3. Cross sections for photodetachment into an $(l, m)=(1,-1)$ state in a $1.07 \mathrm{~T}$ magnetic field (solid curve) and in zero field (dashed curve), as functions of frequency of incident light.

threshold when $\beta_{l}>0$, but are missing altogether when $\beta_{l}<0$. On the other hand, the structures above the thresholds are virtually independent of the sign of $\beta_{\ell}$. This is a familiar feature of single-channel s-wave scattering in the usual 3D situation. What is essential in the case ilustrated by Figs. 1 and 2, however, is the even symmetry of the final-state wave function with respect to reflection in the $x y$ plane (guaranteed whenever $|m|=l$ ), rather than the rotational symmetry at small $r$.

Fig. 3 gives the cross section for photodetachment into an $(\ell, \mathrm{m})=(1,-1)$ state, calculated from Eq. (3). (As above, $\ell$ has a definite meaning only at small r.) An example is $\mathrm{H}^{-}$neutralization by a circularly polarized light beam propagating parallel to $\mathrm{H}$. To strengthen this connection, the constant $\alpha$ was given the value ${ }^{14} 871$ a.u. appropriate to $\mathrm{H}^{-}$. However, a problen arises in assigning the p-wave scattering length $a_{1}$ for $e-H$ scattering. Because the potential falls of only as $r^{-4}$ at large $r$, due to the polarizability, the p-wave scattering length cannot be def ined. For the calculation, a value of unity was chosen for this quantity. The figure resembles the one. Fig. 1. for detachment into an $s$ wave, in having a series of what appear (at $\mathrm{GHz}$ resolution) to be sharp spikes, separated by the cyclotron frequency. There are some differences, however. The zero-field cross section (dashed line) 


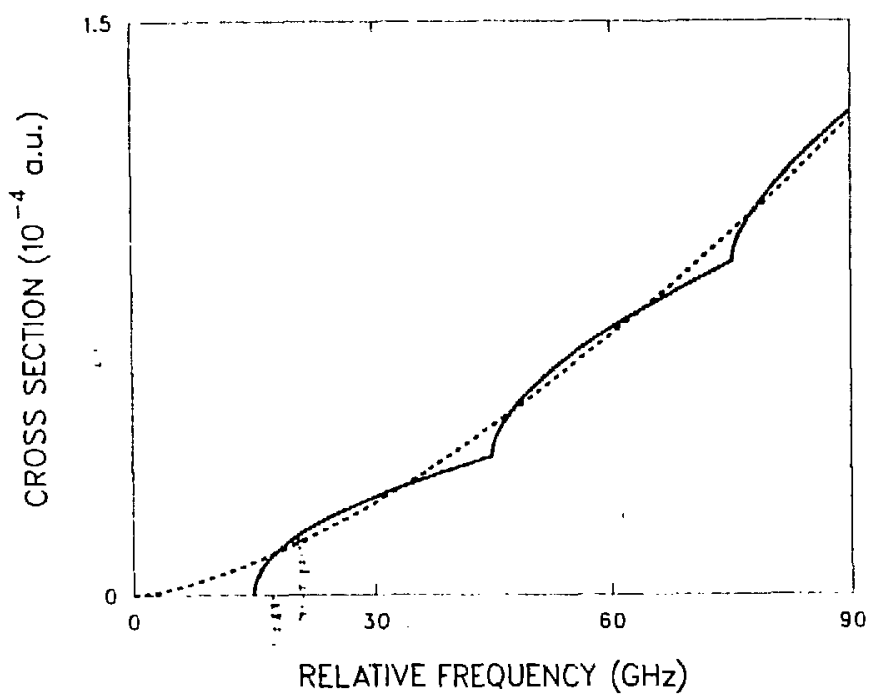

FIG. 4. Cross sections for photodetachment into an $(\ell, \mathrm{m})=(1.0)$ state in a $1.07 \mathrm{~T}$ magnetic field (solid curve) and in zero field (dashed curve), as functions of frequency of incident light.

has a different shape. Still, ${ }_{\sigma}^{\mathrm{H}}$, which oscillates about $\sigma$, has approximately the same value, when averaged over an $\omega_{\mathrm{H}}$ interval, as $\sigma$. The other difference, apparent from Eqs. (2) - (4), is that the near-threshold maxima are located much closer to thresholds when $\ell=1$.

Now consider the case where the final states are characterized by $|m|=\ell-1$. Here, the final-state wave functions have odd symmetry with respect to reflection in the xy plane. We find ${ }^{11}$ that the photodetachment cross section for $(\ell, \mathrm{m})=(1,0)$ is given by

$$
\sigma^{H}(\mathrm{knO})=3 \sqrt{2} a \omega_{\mathrm{H}}^{3 / 2}\left[\frac{\omega-\omega_{\mathrm{mn}}}{\omega_{\mathrm{H}}}\right]^{1 / 2}, \iota=1
$$

We have assumed that neither bound nor virtual states of odd symmetry occur very close to threshold wher $H=0$. They will not be created when the field is applied. Therefore the large features seen abcve where $|m|=l$ would not be expected here, and Eq. (7) shows that such is the case. A plot of $\sigma^{\mathrm{H}}$ for photodetachment from $\mathrm{H}^{-}$in a $1.07 \mathrm{~T}$ field by a laser polarized in the $\mathrm{z}$ direction $(\alpha=871 \mathrm{a} . \mathrm{u} ., \mathrm{m}=0)$ is shown in Fig. 4. One can see that al though $\sigma^{\mathrm{H}}$ increases monotonically, staying close to $\sigma$, the thresholds are clearly evident. 
Detailed comparison with existing experimental data is not possible because of broadening of spectroscopic features. due mainly to thermal motion. Nonetheless, Larson and Stoneman were able to find an indication. in their recent $S^{-}$experiment. ${ }^{10}$ that the peaks are shifted upward in frequency compared with the locations of the Landau level thresholds, in qualitative agreement with a proposal by Clark. ${ }^{7}$ These shifts are orders of magnitude greater than expected from present theory (cf. Fig. 2). (Indeed, at the level of resolution achieved so far, predictions of the theory discussed here for the $m= \pm \ell$ case: where the large oscillations occur, are experimentally indistinguishable from the results of the theory of Blumberg and coworkers.) Furthermore, Larson and Stoneman $f$ ind that the data taken with $\pi$ polarization tend to $f$ it the model better than those taken with $\sigma$ polarization. These findings suggest that a closer examination of the theoretical treatment of transitions between fine structure components would be useful.

$$
\because \text { CONCLUSIONS }
$$

Experiments at higher resolution are needed ${ }^{2}$ to provide critical tests of the theory of photodetachment in a magnetic field. In the meantime, the theory requires further development. There is already a suggestion that it be reexamined, particularly where transitions between fine structure components of ion and atom are involved. ${ }^{10}$ Furthermore, the theory requires extension to cover cases where the scattering length in the exit channel is undefined, as in photodetachment from $\mathrm{H}^{-}$.

\section{REFERENCES}

1. A. I. Hershcovitch and E. A. Hinds, Proc. 3rd Int. Symp. on Production and Neutralization of Negative Ions and Beams, K. Prelec, Editor, Brookhaven National Laboratory, AIP Conf. Proc. No. 111 (1983) p. 763; A. Hershcovitch. Nucl. Instr. Methods in Phys. Research A243, 271 (1986).

2. H. F. Krause, "A Novel High-Resolution Experimental Approach for Studying Laser Photodetachment in a Magnetic Field." this conference.

3. W. A. M. Blumberg, R. M. Jopson, and D. J. Larson, Phys. Rev. Lett. 40, 1320 (1978).

4. W. A. M. Blumberg. W. M. I tano, ant D. J. Larson, Phys. Rev. B19. 139 (1979).

5. L. D. Landau and E. M. Lifshitz, Quantum Mechanics: NonRelativistic Theory. 3rd Ed. (Addison-Wesley, Reading Mass.. 1977). p. 456.

6. D. J. Larson and R. Stoneman, J. Phys. Supp. 11, 43, C2-285 (1982).

7. C. W. Clark, Phys. Rev. A28, 83 (1983). 
8. W. P. Reinhardt, J. Phys. B: At. Mol. Phys. 16. L635 (1983).

9. Yu. A. Gurvich and A. S. Zil 'bermints, Sov. Phys. JETP 58, 754 (1983).

10. D. J, Larson and R. Stoneman, Phys. Rev. A31, 2210 (1985).

11. 0. H. Crawford, to be published.

12. Al though the angular momentum is not well defined in the field. the assumptions made allow us to treat the wave function at small $r$ as though it had a definite value of $\ell$.

13. Atomic units, a.u. $\left(e=m_{e}=h=1\right)$, are used except where otherwise stated.

14. A. L. Stewart, J. Phys. B11. 3851 (1978). 


\section{DISCLAIMER}

This report was prepared as an account of work sponsored by an agency of the United States Government. Neither the United States Government nor any agency thereof, nor any of their employees, makes any warranty, express or implied, or assumes any legal liability or responsibility for the accuracy, completeness, or usefulness of any information, apparatus, product, or process disclosed, or represents that its use would not infringe privately owned rights. Reference herein to any specific commercial product, process, or service by trade name, trademark, manufacturer, or otherwise does not necessarily constitute or imply its endorsement, recommendation, or favoring by the United States Government or any agency thereof. The views and opinions of authors expressed herein do not necessarily state or reflect those of the United States Government or any agency thereof. 